https://creativecommons.org/licenses/by/4.0/

\title{
REFERENTES MEDIO AMBIENTALES PARA LA GESTIÓN DE PROYECTOS EN LAS ORGANIZACIONES
}

\section{Environmental references for the management of projects in organizations}

\author{
WILSON JAVIER CASTRO TORRES ${ }^{1}$, IVONNE EDITH CASTRO TORRES ${ }^{2}$, ANGIE MARIAN GONZÁLEZ GARCÍA ${ }^{3}$ \\ Recibido:30 de mayo de 2017. Aceptado:07 de junio de 2017 \\ DOI:http://dx.doi.org/10.21017/rimci.2017.v4.n8.a34
}

\begin{abstract}
Resumen
El presente artículo realiza una reflexión teórica sobre los elementos conceptuales abordados desde cuerpos de conocimiento como los promovidos por organizaciones y agremiaciones de índole internacional como el Project Management Institute (PMI $($ ) y el Green Project Management (GPM®) como buenas prácticas para la gestión de proyectos, que a su vez, se materializan organizacionalmente con el uso de técnicas y herramientas gerenciales, y como estas definen un comportamiento organizacional con un enfoque hacia la gestión efectiva y estratégica de proyectos, pero que es su quehacer dejan de lado elementos de valoración del uso y oportunidad de los bienes ambientales que son explotados a la hora de ejecutar iniciativas de inversión mediante proyectos y no son incluidos en una evaluación ex - ante ni valorados dentro de la estimación de la línea base del proyecto.
\end{abstract}

Palabras clave: gestión de proyectos, evaluación ambiental, línea base, buenas prácticas, economía ambiental.

\begin{abstract}
This article presents a theoretical reflection on the conceptual elements approached from bodies of knowledge such as those promoted by international organizations and associations such as the Project Management Institute (PMI $)$ and the Green Project Management (GPM®) as better practices for the project management, which in turn, materialize organizationally with the use of techniques and tools managerial, and how these define an organizational behavior with a focus towards the effective and strategic project management, but that is their task leave aside valuation elements the use and timing of environmental assets that are exploited when implementing investment initiatives through projects and are not included in an ex ante evaluation or valued within the project baseline estimate.
\end{abstract}

Keywords: project management, environmental assessment, baseline, good practices, environmental economics.

\section{INTRODUCCIÓN}

E diferentes puntos de vistas epistemológicos, sin embargo, de acuerdo a Sisk y Sverdlik se define como un conjunto de acciones y funciones realizadas por empresarios, gerentes o supervisores, e incluso otros lo refieren a un grupo particular de personas es sinónimo del ejercicio de autoridad sobre sus vidas de trabajo [1]; en resumen, la gerencia incluye los procesos de planear, organizar, actuar y controlar las diferentes actividades y operaciones de la organización, que por medio de la coordinación del talento humano y los diferentes recursos, lograr los objetivos de forma efectiva. El término gerencia puede describir como una actividad realizada por un profesional de planear, dirigir y alcanzar los objetivos de la organización con la integración y aprovechamiento de los recursos asignados; es decir hace alu-

1 Docente Investigador de la Corporación Universitaria Republicana., vinculado al grupo de investigación OCA. Correo electrónico: wcastro@ urepublicana.edu.co

2 Docente Investigadora Universidad Piloto de Colombia, vinculada al grupo de investigación IG. Correo electrónico: ivonne-castro@ unipiloto.edu.co

3 Estudiante facultad de ingeniería - Semillero de investigación en Uso y aplicación de métodos cuantitativos en la gestión empresarial - Grupo de investigación OCA 
sión a la labor o función de colocar en práctica los métodos, técnicas, estándares conocimientos y experiencias que permitan el mejor funcionamiento de la empresa u organización. Como lo señala Gutiérrez, los proyectos se transforman en la herramienta para lograr los objetivos estratégicos que se desarrollan mediante una sinergia del colectivo organizacional [2] en sincronía con estos elementos el PMI® establece que la gestión de proyectos es una herramienta que ejecuta la estrategia empresarial mediante el manejo efectivo de aspectos técnicos, administrativos, pero además de ello de elementos particulares de cada proyecto determinados por las variables de entorno y las expectativas de los stakeholders [3], conforme a lo anterior aparecen un conjunto de técnicas y herramientas que permiten realizar una gestión efectiva del proyecto dentro de las fases de inicio, planificación, ejecución, monitoreo y cierre, esta aplicación eficiente de estos recursos gerenciales impactan en el éxito del proyecto, tal como lo señalan los resultados del Pulse of the Profession ${ }^{\circledR} 2015$ que revelan que las organizaciones de alto desempeño respaldan la dirección y gestión de proyectos a lo largo de la organización y que además de estar alineados con la estrategia empresarial, gestionan el talento de sus colaboradores y aplican prácticas estandarizadas de dirección y gestión de proyectos en toda la organización [4]. En ese sentido se logra evidenciar una armonía de los planteamientos que aterrizan la gerencia de proyectos, sin embargo la inclusión de elementos que aporten a la evaluación de impacto relacionado con el medioambiente en muchas ocasiones se subvaloran por parte de la gerencia de la organización y la misma dirección del proyecto.

El presente documento tiene como propósito establecer los elementos de común aplicación en el marco de lo establecido como buena practica desde la perspectiva del PMI ${ }^{\circledR}$ y el GPM® para la gestión de proyectos y determinar cómo estos, son aplicables para la medición e inclusión de variables de valoración del impacto ambiental causado por el uso de bienes ambientales que son explotados durante la fase de ejecución del proyecto, para con ello establecer elementos de partida para la vinculación dentro de las practicas organizacionales técnicas y herramientas que propendan por el desarrollo sostenible y la conservación de los recursos naturales.

\section{Referentes Conceptuales}

El abordaje de la medición, uso, aplicación y adopción de prácticas estandarizadas permite a su vez el incremento del valor agregado generado con un proyecto; sin embargo, a la luz de las prácticas estandarizadas, se pueden encontrar algunos referentes en Colombia como un estudio realizado en 226 empresas bogotanas en el año 2010 se concluye que cada unidad de negocio de estas organizaciones desarrolla y aplican su propia metodología para la dirección y gestión de proyectos y estas tienen la falencia que no son estandarizadas, no incorporan procesos, herramientas y técnicas derivadas de buenas prácticas y estándares internacionales [5], entre esas buenas practicas aparece una nueva corriente hacia el desarrollo de proyectos con enfoque sustentable promovida por el Green Project Management ${ }^{\circledR}$ mediante el desarrollo de la metodología PRisMTM $®$, que consiste en una metodología de gestión de proyectos sostenible basada en principios, donde la principal diferencia con respecto a los enfoques tradicionales es que incorpora un modelo de maximización del valor que se centra en el ciclo de vida total del activo [6], es decir incorpora dentro de la fase de planificación del ciclo de vida del producto elementos valorativos del ciclo de vida del producto generado por el desarrollo del proyecto y lo transforma en un modelo efectivo de gestión de proyectos que alcanza los objetivos estratégicos de la empresa, enfatizando la responsabilidad con el entorno, e incorpora modelos de medición del impacto ambiental generado por el desarrollo y puesta en marcha de un proyecto en sincronía con los elementos de valoración e indicadores definidos por el programa de las naciones unidas para el medio ambiente y el desarrollo PNUMAD, y la comisión para el desarrollo sostenible CDS, que como lo señala Labandería, existe la necesidad de definir indicadores para que el concepto de sustentabilidad alcanzase a ser algo más que un objetivo difuso, y así desarrollar indicadores de desarrollo sostenible para proveer de bases sólidas a la toma de decisiones a todos los niveles y para contribuir a una sostenibilidad autorregulada y realizar un aporte desde el desarrollo económico que permita llegar a un consenso en que debe incorporar objetivos económicos, ambientales y sociales [7], entonces la empresa como ente que pertenece y realiza un aporte a la construcción y desarrollo de la sociedad demanda la integración 
de prácticas que propendan por el desarrollo de actividades en un marco de sostenibilidad que permitan la medición y control de los impactos ambientales causados por el desarrollo de sus actividades empresariales y una de estas es el desarrollo de proyectos.

\section{LA GESTIÓN DE PROYECTOS Y LA GESTIÓN AMBIENTAL}

La gestión de proyectos ha cobrado una papel importante en el desarrollo económico del país más aun cuando la ejecución de la política pública y la asignación de fondos públicos para inversión se realiza conforme al conjunto de programas desplegados desde el plan de gobierno y ejecutados por el departamento de planeación nacional mediante la aplicación de la metodología general ajustada que tiene su fundamento en la metodología general para la identificación, formulación y evaluación de proyectos promovida por la CEPAL ${ }^{4}$ que presenta de forma clara enfoques para solucionar problemas; análisis de involucrados; participación ciudadana; incorporación del enfoque género en el ciclo de los proyectos; incorporación de la variable ambiental [8]; sin embargo, es esta última variable la que se valora de manera superflua a la hora de preparar los planes de gestión de proyecto, puesto que la normativa ambiental colombiana establece una serie de criterios de inclusión como estrategias de mitigación y evaluación del impacto ambiental a la hora de desarrollar el proyecto pero deja de lado la valoración e inclusión dentro de los costos del proyecto y su presupuesto los elementos que hacen referencia al costo de oportunidad y costo de uso de los bienes ambientales razón por la cual la estimación presupuestal y asignación de fondos no contempla los aspectos inherentes al consumo y explotación de recursos ambientales que si bien es cierto no po- seen un mercado en el que se puedan tranzar si existen metodologías que permiten determinar su valor monetario esperado.

Claro ejemplo de esta situación se encuentran los proyectos financiados por el Banco interamericano de desarrollo ${ }^{5}$ quien cofinancia iniciativas de inversión articuladas dentro del plan de desarrollo del gobierno nacional y otros de gran interés desarrollados por iniciativa pública en cumplimiento del plan de gobierno entre los que se destacan según Bnamericas ${ }^{6}$ autopista de la prosperidad con una inversión cercana de los 7.170 MUSD, Ferrocarril del Casanare por 2.500 MUSD, Líneas del tren ligero de Bogotá 895 MUSD [9], sin embargo, aunque las cifras son elevadas solo representan una proporción de los recursos que se invierten en la ejecución de proyectos de inversión privada que alcanzan solamente en la ciudad de Bogotá según Ómar Oróstegui, director de Bogotá Como Vamos, la cifra de 2.800 MUSD; por otro lado en telecomunicaciones se proyectan inversiones por US $\$ 3.300$ millones, de las cuales el $35 \%$ corresponderá al sector privado, por el lado de carreteras la inversión llegará a los US\$900 millones, mayoritariamente privada, en energía, la participación privada es ya de gran importancia con proyectos como el de Proeléctrica en Mamonal con una inversión de US\$56 millones y la Planta Térmica de Las Flores en Barranquilla de un consorcio colombo - español por US\$150 millones [10], las anteriores iniciativas que si bien es cierto realizan una contribución importante al desarrollo económico y social del país causan un efecto adverso al medio ambiente y los recursos naturales como lo reconoce el anterior ministro de medio ambiente y desarrollo sostenible Neider Eduardo Abelló al referir que la política ambiental en Colombia ha evolucionado satisfactoriamente pero hace falta reforzar esfuerzos en esquemas de control y gestión ambiental [11].

4 Comisión económica para el desarrollo de América Latina y el Caribe, La CEPAL es una de las cinco comisiones regionales de las Naciones Unidas y su sede está en Santiago de Chile, e fundó para contribuir al desarrollo económico de América Latina, coordinar las acciones encaminadas a su promoción y reforzar las relaciones económicas de los países entre sí y con las demás naciones del mundo. https:// www.cepal.org/es

5 Banco interamericano de desarrollo (BID) El BID es la principal fuente de financiamiento multilateral de América Latina. Ofrece soluciones para afrontar los retos del desarrollo y apoyo en áreas claves de los gobiernos adscritos a las naciones unidas, es posible consultar las iniciativas de proyectos aprobadas en: http://www.bancomundial.org/es/country/colombia/projects/all

6 BNamericas. Business News Americas (BNamericas), es una fuente de inteligencia de negocios enfocada en América Latina. https:// web5.bnamericas.com/bnamericas/landing/about-us/index-es.html 
La política pública ambiental en Colombia tiene sus inicios en 1991, con la constitución política de Colombia que establece en su artículo 79 al medio ambiente como derecho fundamental [12], compromiso que se reafirma más adelante en 1993 con la ley 99, por el cual se crea el Ministerio del Medio Ambiente, se reordena el sector público encargado de la gestión y conservación del Medio Ambiente y los Recursos Naturales Renovables, se organiza el Sistema Nacional Ambiental [13]. Dentro de las reglamentaciones establecidas por el estado encontramos los informes de cumplimiento ambiental ("ICA") en el cual se incluyen la obligatoriedad de generar estudios de impacto ambiental ("EIA") y los planes de manejo ambiental ("PMA") que contemplan entre otras cosas la línea base antes de la actividad y su relación directa con posibles contaminantes. No obstante la perspectiva de este tipo de informes no engloba el concepto amplio que se debe trabajar de medioambiente $y$ se reduce a generar panoramas del proyecto desde lo ecológico y ambiental.

De otra parte, se evidencia que más allá de las instituciones la puesta en marcha de la política pública como lo menciona el departamento de políticas públicas y descentralización de Chile; la ejecución de la política se materializa luego de una evaluación ex ante que permita formular y desarrollar planes y programas de desarrollo regional [14], comprendiendo esto se genera el primer plan nacional de desarrollo forestal el cual tiene como objetivo establecer un marco estratégico que incorpore el sector forestal al desarrollo nacional a partir del manejo sostenible de los bosques naturales y plantados [15], elemento que se transforma en la punta de lanza para determinar restricciones en la formulación y desarrollo de proyectos de inversión pública o privada que tengan impactos sobre el ecosistema, hasta llegar a la consolidación de política nacional para humedales interiores, estrategias para su conservación y uso racional, que permite consolidar el compromiso estatal de propender por la conservación y el uso racional de los humedales interiores de Colombia con el fin de mantener y obtener beneficios ecológicos, económicos y socioculturales, como parte integral del desarrollo del país [15].

Estos dos instrumentos parten del criterio de evaluación de la eficacia ambiental, vinculando los conceptos de efectividad ambiental definido como la capacidad del instrumento para solucionar un problema ambiental, la incentivación a la introducción y desarrollo de tecnologías limpias [7] y la eficiencia económica como principio de la economía en que los gobiernos pueden mejorar los resultados del mercado a través de políticas que buscan controlar los precios porque consideran que los resultados del mercado son injustos [16] y a través de la intervención del estado aproximarse a una solución óptima que permita encontrar el punto de equilibrio entre el costo marginal de consumir y explotar bienes ambientales y el costo de oportunidad ligado a ese bien mediante su conservación; de hecho Guhl Nanetti refiere que la política ambiental en Colombia está desarrollada y avanzada pero el problema es que no se ejecuta, señalando que hay un Sistema Nacional Ambiental que existe jurídicamente, pero que no se ha materializado en la realidad [17].

Ante este panorama el compromiso del gobierno nacional decretado en el plan de desarrollo Prosperidad para Todos (2010-2014) y todos por un nuevo país (2014-2018) establece el derrotero en materia ambiental que permitirá alcanzar y ratificar el compromiso ambiental decretado por el gobierno nacional [18] en la ratificación del acuerdo de París donde el país se compromete a reducir su emisión de gases efecto invernadero en un $20 \%$ y con ello desarrollar una de las seis estrategias en materia ambiental 'Crecimiento verde' que busca mejorar la salud ambiental para disminuir del $2 \%$ a $1,7 \%$ los costos en la salud asociados a la contaminación del aire y los sistemas inadecuados de agua, saneamiento e higiene como porcentaje del PIB [19].

Es así como una deficiencia en la ejecución de la política pública por parte de los entes reguladores y la institucionalidad del estado representada por el ministerio del medio ambiental y desarrollo sustentable y las corporaciones autónomas regionales demanda la inclusión y compromiso por parte de los actores privados para la contribución a estos ambiciosos planes en tema medio ambiental, es así como no solo en la actividad productiva sino en el los programas planes de inversión se incluyen estos elementos como criterios de aceptación y validación para el desarrollo y ejecución de proyectos; es decir el papel estatal se limita al uso de instrumentos vía comando, control y gestión ambiental en materia

Rev. Ingeniería, Matemáticas y Ciencias de la Información Vol. 4 / Núm. 8 / julio-diciembre de 2017; pág. 69-75 
de los permisos de vertimiento, control, seguimiento y multas, que define parámetros vía precios de mercado como impuestos por vertimientos, impuesto por afectación de la calidad del aire entre otros para el desarrollo de actividades que demanden inversión y tengan una afectación al medio ambiente, elemento que es señalado por Labanderia como mecanismos de precio más habituales que tienen el objetivo de recoger el daño ambiental causado por las emisiones gravadas [7].

Entonces, bajo esta premisa será el papel del gerente de proyecto y el comité gerencial de los proyectos definir esquemas de control ambiental que no solo se resuman en la actividad de verificación y auditoria de actividades, sino que tengan una presencia desde la formulación y conceptualización del proyecto de tal modo que permitan la inclusión de buenas prácticas ambientales en proyectos que incorporen un modelo de maximización del valor que se centra en el ciclo de vida total del active [6], es decir dentro de la fase de planificación del ciclo de vida del producto elementos valorativos del ciclo de vida del producto, que permita cuantificar y transformar en variables económicas las acciones que el equipo de proyecto debe ejecutar para mitigar el impacto ambiental y con ello sacar el mayor provecho desde el punto de vista de los costos económicos del proyecto maximizando el valor agregado del proyecto vía costos no desembolsados que se generan por la reducción del impacto ambiental generado por las actividades propias del proyecto y medidas desde los parámetros que establece la legislación ambiental y tributaria del gobierno nacional.

Ante ese panorama los cuerpos de conocimiento promovidos por el Green Project Management y el Project Management Institute establecen elementos y criterios de aplicación para una gestión efectiva de un proyecto dentro del ciclo de vida del proyecto y del producto, partiendo de esto se establece una batería de herramientas que el gerente de proyecto debe aplicar dentro de la gestión de las áreas de conocimiento definidas por el PMBoK y siendo de esta manera incluso se desconoce dentro de estas la gestión ambiental al interior del proyecto y lo traslada a una fase preliminar, esto no quiere decir que no existe una preocupación por la inclusión de elementos de gestión y valoración ambiental incluso estos se pueden evidenciar en el construction extention
Guide ${ }^{\circledR}$ una guía a la construcción específica para el profesional de gestión de proyectos para cada una de las áreas de conocimiento, así como la orientación en áreas adicionales que no se encuentran en el PMBOK ${ }^{\circledR}$ como lo son gestión de recursos, salud, seguridad, seguridad y gestión ambiental, la gestión financiera del proyecto, además de la gestión de reclamaciones [20].

Por otro lado el Green Project Management establece criterios de valoración en pro de la triple línea base de sostenibilidad (social, ambiental y rentabilidad) mediante un cuerpo de conocimiento definido por el estándar P5 ${ }^{\mathrm{TM}}$ de sostenibilidad para la gestión de proyectos que valora la gestión del proyecto en 5 elementos las personas, el planeta, la rentabilidad, los procesos de gestión y los productos, ahora pese a estos esfuerzos en términos de elementos vinculantes y necesarios para la aplicación de conocimientos en pro de culminar un proyecto de forma exitosa dentro de las restricciones de alcance, tiempo, costo y calidad respetando las restricciones de los interesados, aparece la normativa ambiental colombiana que establece que para el desarrollo de un proyecto es necesario la expedición de una licencia ambiental, amparada dentro del decreto 2820 de 2010 donde se establece que el alcance de un proyecto establece todas las actividades de planeación, emplazamiento, instalación, construcción, montaje, mantenimiento, operación, desmantelamiento, abandono y terminación de todas las acciones y uso de espacio [20], razón por la cual dentro del ciclo de vida del proyecto es necesario establecer acciones que permitan prevención, mitigación, corrección, compensación e incuso manejo de los efectos ambientales causados que pueden ser desde la modificación hasta el deterioro de los recursos ambientales.

Estos requerimientos fueron validados mediante el decreto 2014 de 2014 por el cual se reglamenta la expedición y competencia de las licencias ambientales y da potestad a las entidades territoriales, corporaciones autónomas regionales y a la autoridad nacional de licencias ambiental para la gestión y control sobre la expedición de estos permisos que regulan las actividades $\mathrm{n}$ los proyectos, entonces teniendo $\mathrm{n}$ panorama normativo claro, se evidencia la falencia a la hora de aterrizar estos requerimientos en procesos de gerencia sistemáticas que incluya la valoración y medición de variables ambientales dentro de las actividades propias 
de la gestión del cronograma, gestión del tiempo y riesgos que permiten validar y actualizar la línea base del proyecto.

\section{CONCLUSIONES}

Los proyectos demandan un conjunto de esfuerzos gerenciales y administrativos de diferente nivel de alcance dependiendo de la fase de desarrollo en la que se encuentre, sin embargo es necesario la inclusión de procesos estandarizados con herramientas y técnicas propias de una cultura organizacional proyectizada, sin embargo en pro de cumplir con los estamentos normativos y dar cumplimiento a una gestión sustentable que propenda por el bienestar social y la conservación de los recursos naturales es necesario que dentro de la cultura organizacional se realice un abordaje profundo de un enfoque de economía ambiental que ermita valorar el uso de los recursos naturales e incluya los costos de uso y oportunidad de los bienes ambientales dentro de la estimación, medición y control de los componentes de la línea base del proyecto.

El concepto medioambiente debe ser abordado de manera amplia, es decir su construcción debe incluir aspectos relacionados con lo ambiental, lo cultural y lo social en atención a premisas de desarrollo territorial sustentable, esto con el único fin de lograr un análisis sobre vacíos y debilidades del proyecto que genere un abordaje integral de riesgos para la respectiva mitigación y compensación los posibles impactos que se generen.

Los cuerpos de conocimiento con reconocimiento internacional aparecen con instrumento que usa el formulador o gerente de proyecto para el desarrollo de las iniciativas de inversión, sin embargo y pese al esfuerzo realizado por los cuerpos colegiados que dirigen estas iniciativas no es lo suficientemente integral para abordar todas las dimensiones y aristas económicas y de contexto que puede tener un proyecto, es por ello que el gerente o evaluador debe estar en a capacidad de aplicar un dosier de elementos que permitan garantizar el éxito del proyecto tratando de preservar en mayor medida los recursos ambientales, teniendo en cuenta que la integración de elementos ambientales dentro del ciclo de vida del proyecto no es abordará de manera explícita dentro de estos.

\section{REFERENCIAS}

[1] J. P. Perez, «Instituto Universitario de Tecnologia». 1998.

[2] S. Gutierrez, y K. Vargas \& S. Gracia, «Proyectos, Innovación y Estrategia. Un paso firme hacia nuevos modelos en la gestión empresarial» Tecnología en marcha, 69-84. 2011.

[3] PMI, P. M. «Guia de los fundamentos para la dirección de proyectos», PMBoK. Pennsilvania. 2016.

[4] Project Management Institute. «Pulse of profession. Pennsilvania». 2015.

[5] S. Arce \& H. Lopez, «Valoración de la gestión de proyectos en empresas de Bogotá». Revista EAN, 60-87. 2010.

[6] Green Project Management. «PRisM», Proyectos integradores de mètodos sustentables. Obtenido de https:/ / greenprojectmanagement.org/ prismmethodology. 2017.

[7] A. Labandería, C. Leon, \& M. Vasquez. «Economía Ambiental». Madrid: Pearson Education. 2007.

[8] CEPAL. Metodología general para la identificación, preparación y evaluación de proyectos de inversión pública. Santiago de Chile: Publicaciones de las naciones Unidades. 2005.

[8] J. Detonni, «BNamericas». Obtenido de Los 10 principales proyectos de infraestructura de Colombia - BNamericas 3rd Andean Infrastructure Summit: https://www.bnamericas.com/es/features/infraestructura/los-10-principales-proyectos-deinfraestructura-de-colombia-bnamericas-3rdandean-infrastructure-summit.2013.

[9] DINERO. «Revista Dinero». Obtenido de Proyectos públicos con capital privado: http://www.dinero. com/edicion-impresa/negocios/articulo/proyectos-publicos-capital-privado/20459?utm_source= semana.com\&utm_medium =referral\&utm campaign=otras-publicaciones-busqueda. 2012.

[10] N. Abello, «Valoración de bienes y servicios ambientales como herramienta para diseño de instrumentos de gestión ambiental en Colombia». Bogotá, Colombia. Obtenido de https://www.youtube. com/watch?v=6bqmpDQq1y4. 2013.

[11] Republica de Colombia. «Constitución politica». Bogotá. 1991.

[12] Republica de Colombia. «Ley 99, Sistema nacional ambiental». Bogotá. 1993.

[13] SUBDERE. «Guía Metodológica para la formulación de politicas públicas regionales». Santiago de Chile, Chile: Subsecretaria de desarrollo regional y administrativo. 2009.

Rev. Ingeniería, Matemáticas y Ciencias de la Información Vol. 4 / Núm. 8 / julio-diciembre de 2017; pág. 69-75 
[14] Banco de la Republica. «Política ambiental colombiana». Bogotá. 2015.

[15] G. Mankiv, «Principios de economía». Mexico D.F: Cengage Learning. 2012.

[16] Semana «La política ambiental de Colombia es avanzada, el problema es que no se pone en práctica». Semana Sostenible. 2015.

[17] Republica de Colombia. «Plan nacional de desarrollo (2014- 2018)» Todos por un nuevo país. Bogotá: Direcciòn nacional de planeción. 2014.
[18] DINERO. «Este es el aporte ambiental al plan nacional de desarrollo». Dinero. 2015.

[19] Project Management Institute. "Construction Extention Guide». Netsquare, Pensilvania. 2012.

[20] Ministerio del medio ambiente, vivienda y desarrollo territorial, 2010. 
